\title{
A Função Barreira Modificada e o Problema de Fluxo de Potência Ótimo ${ }^{1}$
}

E.C. BAPTISTA2, Departamento de Matemática, FC, UNESP, 17033-360 Bauru, SP, Brasil

V.A. SOUSA 3 , G.R.M. COSTA, Departamento de Engenharia Elétrica, EESC, USP, 13566-590 São Carlos, SP, Brasil.

Resumo. Uma nova abordagem para a resolução do problema de Fluxo de Potência Ótimo é apresentada. Fazemos uso de pesquisas recentes, especialmente na área dos métodos de pontos interiores. Nesta abordagem, as restrições de igualdade são tratadas pelo método de Newton e as de desigualdade pelo método de Barreira Modificada. Os testes numéricos, mostram o efetivo desempenho desta metodologia.

\section{Introdução}

As funções Barreira foram estudadas inicialmente por Frisch [5] e por Carrol [2], porém o método de Barreira foi realmente popularizado por Fiacco e McCormick [4]. Os autores que trabalharam com o método da função Barreira perceberam que ele apresenta alguns problemas, tais como: o mal condicionamento da matriz Hessiana quando seu fator de Barreira tende a zero; a dificuldade na escolha do fator de Barreira e na escolha de uma solução inicial; a não-existência da derivada na solução e o aumento ilimitado da função Barreira na vizinhança da fronteira. Em virtude desses problemas, na década de 70, o entusiasmo no uso da função Barreira diminuiu sensivelmente. O interesse pelo método da função Barreira reapareceu somente após a apresentação feita por Karmarkar [7], em 1984, de seu método projetivo para Programação Linear. Em 1992, Polyak [8] desenvolveu uma teoria de métodos da função Barreira Modificada. Estes métodos combinam as melhores propriedades da função Lagrangiana Clássica e da função Barreira Clássica, evitando os problemas que ambas enfrentam. Por exemplo, em contraste com a função Barreira Clássica, as funções Barreira Modificadas são definidas na solução; são suaves na vizinhança do ótimo e não vão para o infinito quando o ótimo se aproxima. Neste trabalho propomos a aplicação da função Barreira Modificada ao problema de Fluxo de Potência Ótimo (FPO). O FPO é um problema de otimização não-linear, estático, o qual calcula um conjunto de variáveis ótimas de estado da rede, a partir de dados

\footnotetext{
${ }^{1}$ Trabalho financiado pela FAPESP e CNPq.

${ }^{2}$ baptista@fc.unesp.br

3 vsousa@sel.eesc.usp.br

${ }^{4}$ geraldo@sel.eesc.usp.br
} 
de carga e dos parâmetros do sistema. O problema de FPO otimiza uma função objetivo, a qual pode ser: custo de geração, perdas ativas na transmissão, entre outras; sujeito a restrições de igualdade e desigualdade. As restrições de igualdade representam as equações não-lineares do fluxo de potência e as de desigualdade os limites de geração de potência ativa e reativa, magnitude das tensões nas barras, tap nos transformadores, fluxos de potência ativa e reativa nas linhas de transmissão e transformadores, e as potências de intercâmbio entre áreas.

O problema de FPO foi proposto por Carpentier [1], no início da década de 60, a partir do problema de Despacho Econômico (DE). Historicamente, o problema de $\mathrm{DE}$, resolvido pelo método dos custos incrementais iguais, foi o precursor do problema de FPO, o qual marcou o fim do período clássico do DE. Desde então, muitos trabalhos foram desenvolvidos na tentativa de resolvê-lo. Recentemente, o desempenho dos algoritmos de pontos interiores, como pode ser visto em [11] e [9], tem motivado a sua aplicação na resolução do problema de FPO. Em [6] e [10] um algoritmo Primal-Dual Barreira Logarítmica foi aplicado na resolução do problema de FPO e as condições de Karush-Kuhn-Tuker (KKT) foram satisfeitas utilizando o método de Newton, sendo a maior dificuldade do algoritmo a escolha do parâmetro de barreira. Em [3] foi proposto a resolução do problema de FPO utilizando o método de Newton associado à função Lagrangiana Aumentada. Muitos outros trabalhos foram desenvolvidos utilizando diferentes técnicas de Programação NãoLinear para a resolução do problema de FPO, mas até o presente, não existe uma abordagem, realmente, robusta, confiável e rápida que atenda às necessidades dos centros de operação das concessionárias de energia elétrica. Entre os métodos vistos na resolução do FPO destacamos os métodos de Newton e da Barreira, por apresentar algumas particularidades em sua aplicação. O método de Newton apresenta um excelente desempenho para problemas com restrições de igualdade. Contudo, para restrições de desigualdade, exige-se que se conheça o conjunto ativo na solução. Isso pode dificultar o processo de convergência. No método da Barreira, gerenciar o fator de Barreira e seu parâmetro de atualização, garantindo a convergência, é uma tarefa difícil, porém sua aplicação a problemas com restrições canalizadas é muito vantajosa, pois em todas iterações temos uma solução admissível.

Com o objetivo de aproveitar as características dos métodos citados, apresentamos uma metodologia para resolver o problema de FPO. Esta metodologia trata as restrições de desigualdade através do método da Barreira Modificada as quais são incorporadas à função objetivo, resultando em um novo problema, que é associado à função Lagrangiana. As condições necessárias de primeira ordem são aplicadas resultando num sistema não-linear, que é resolvido pelo método de Newton. A solução através do método de Newton fornece os fatores de correção para atualizarmos as variáveis primais e duais. Os multiplicadores de Lagrange da função Barreira Modificada são atualizados pela regra proposta por Polyak [8] e os fatores de Barreira são atualizados por um fator preestabelecido. 


\section{A Função Barreira Modificada}

A função Barreira Modificada foi proposta por Polyak [8], em 1992, com o objetivo de combinar as melhores propriedades da função Lagrangiana Clássica e da função Barreira Clássica, evitando algumas das dificuldades que ocorrem com ambas. Comparada à função Barreira ela é definida na solução, é suave na vizinhança do ótimo, não vai para o infinito quando o ótimo se aproxima e representa explicitamente os multiplicadores de Lagrange. Além disso, é estritamente convexa na vizinhança da solução quando comparada com a função Lagrangiana Clássica . É considerada como uma função Lagrangiana Aumentada Interior e é utilizada na resolução de problemas restritos. O método da função Barreira Modificada transforma o problema restrito em um irrestrito equivalente, e resolve uma seqüência de problemas irrestritos até atingir o ótimo.

Em seu trabalho, Polyak, apresenta três tipos de função Barreira Modificada: uma para a função Barreira de Carrol, outra para a função Barreira de Frisch e a função Barreira Shift. Neste trabalho utilizaremos a função Barreira Modificada de Frisch, isto é, a logarítmica. Estas funções são definidas através da relaxação do conjunto de restrições factíveis.

Seja o problema

$$
\begin{array}{ll}
\text { Minimizar } & f(x) \\
\text { sujeito a: } & c_{i}(x) \geq 0, i=1, \ldots, m,
\end{array}
$$

onde $x \in R^{n}$. A função Barreira Modificada (FBM) associada ao problema (2.1) é formulada por:

$$
F B M=f(x)-\delta^{-1}\left(\sum_{i=1}^{m} \mu_{i} \ln \left(\delta c_{i}(x)+1\right)\right)
$$

se $x$ pertence ao interior da região factível relaxada, isto é,

$$
\left\{x \in R^{n} \mid c_{i}(x) \geq-\delta^{-1}\right\} .
$$

Minimizando (2.2) em relação à $x, \operatorname{com} \mu_{i}, i=1, \ldots, m$, e $\delta^{-1}$ fixos, temos que satisfazer a seguinte condição

$$
\nabla f(x)-\sum_{i=1}^{m} \frac{\mu_{i}}{\delta c_{i}(x)+1} \nabla c_{i}(x)=0,
$$

que sugere a atualização do multiplicador de Lagrange através da seguinte regra:

$$
\mu_{i}^{K+1}=\frac{\mu_{i}^{K}}{\delta^{K} c_{i}\left(x^{K}\right)+1} .
$$

Na próxima seção utilizaremos a função Barreira Modificada para resolução do problema de FPO. 


\section{A Função Lagrangiana Barreira Modificada e o Problema de Fluxo de Potência Ótimo}

O problema de FPO pode ser formulado como

$$
\begin{gathered}
\text { Minimizar } f(x) \\
\text { sujeito } a: g_{i}(x)=0, i=1, \ldots, m, \\
h_{j}(x) \leq 0, j=1, \ldots, r, \\
x^{\min } \leq x \leq x^{\max },
\end{gathered}
$$

onde $f(x)$ representa as perdas de potência ativa na transmissão; $x^{T}=(\theta, V, t)$ representa o vetor das variáveis de estado do problema; $x^{\max }$ e $x^{\min }$ representam o vetor dos limites superiores e inferiores, respectivamente, das variáveis de estado; $g(x)=0$ representa o conjunto das equações de balanço do fluxo de potência e $h(x) \leq 0$ representa o conjunto das restrições funcionais.

No problema (3.1) acrescentamos às restrições de desigualdade e canalizadas variáveis auxiliares, isto é,

$$
\begin{aligned}
\text { Minimizar } & f(x) \\
\text { sujeito } a: & g_{i}(x)=0, i=1, \ldots, m, \\
& h_{j}(x)+s_{j}=0, j=1, \ldots, r, \\
& x+s u=x^{\max }, \\
& x-s \ell=x^{\min }, \\
& s_{j} \geq 0, j=1, \ldots, r \\
& s u \geq 0 \\
& s \ell \geq 0
\end{aligned}
$$

onde $s u^{T}=\left(s u_{1, \ldots,} s u_{n}\right)$, com $s u_{k} \geq 0$, e $s \ell^{T}=\left(s \ell_{1, \ldots,} s \ell_{n}\right)$, com $s \ell_{k} \geq 0$, para $k=1, \ldots, n$. As variáveis $s_{j}, j=1, \ldots, r$, bem como as componentes do vetor $s u$ são variáveis de folga e as componentes do vetor $s \ell$ são variáveis de excesso.

Adicionamos à função objetivo as condições de não-negatividade, através da função Barreira Modificada e obtemos o problema modificado:

$$
\begin{array}{cl}
\text { Minimizar } & f(x)-\delta^{-1}\left(\sum_{k=1}^{n} \mu u_{k} \ln \left(\delta s u_{k}+1\right)+\right. \\
& \left.\sum_{k=1}^{n} \mu \ell_{k} \ln \left(\delta s \ell_{k}+1\right)+\sum_{j=1}^{r} \mu_{j} \ln \left(\delta s_{j}+1\right)\right) \\
\text { sujeito } a: & g_{i}(x)=0, i=1, \ldots, m, \\
& h_{j}(x)+s_{j}=0, j=1, \ldots, r \\
& x+s u=x^{\max } \\
& x-s \ell=x^{\min }
\end{array}
$$

onde $\delta^{-1}$ é o fator de barreira. 
Associamos ao problema (3.3) a seguinte função Lagrangiana:

$$
\begin{aligned}
& L a(x, s u, s \ell, s, \lambda, \pi u, \pi \ell, \pi)=f(x)-\delta^{-1}\left(\sum_{k=1}^{n} \mu u_{k} \ln \left(\delta s u_{k}+1\right)+\right. \\
& \left.\sum_{k=1}^{n} \mu \ell_{k} \ln \left(\delta s \ell_{k}+1\right)+\sum_{j=1}^{r} \mu_{j} \ln \left(\delta s_{j}+1\right)\right)+\sum_{i=1}^{m} \lambda_{i} g_{i}(x)+ \\
& \sum_{k=1}^{n} \pi u_{k}\left(x_{k}+s u_{k}-x_{k}^{\text {max }}\right)+\sum_{k=1}^{n} \pi \ell_{k}\left(x_{k}-s \ell_{k}-x_{k}^{\text {min }}\right)+\sum_{j=1}^{r} \pi_{j}\left(h_{j}(x)+s_{j}\right)
\end{aligned}
$$

que é denominada função Lagrangiana Barreira Modificada.

Com o objetivo de obtermos a solução do problema (3.1) aplicamos a condição de otimalidade à função Lagrangiana Barreira Modificada (3.4) e obtemos o sistema não-linear:

$$
\nabla L a=0
$$

onde

$$
\nabla L a=\left(\begin{array}{c}
\nabla_{x} f(x)+\lambda^{T} J_{1}(x)+\pi u^{T} I+\pi \ell^{T} I+\pi^{T} J_{2}(x) \\
-\frac{\mu u_{k}}{\delta s u_{k}+1}+\pi u_{k}, k=1, \ldots, n \\
-\frac{\mu \ell_{k}}{\delta s \ell_{k}+1}-\pi \ell_{k}, k=1, \ldots, n \\
-\frac{\mu_{j}}{\delta s_{j}+1}+\pi j, j=1, \ldots, r \\
g_{i}(x), i=1, \ldots, m \\
x+s u-x^{\max } \\
x-s \ell-x^{\min } \\
h(x)+s
\end{array}\right)
$$

$J_{1}(x)^{T}=\left(\nabla_{x} g_{1}(x), \ldots, \nabla_{x} g_{m}(x)\right)$ e $J_{2}(x)^{T}=\left(\nabla_{x} h_{1}(x), \ldots, \nabla_{x} h_{r}(x)\right)$ são denominadas matrizes Jacobianas e I é a matriz identidade.

A solução do sistema não-linear (3.5), é obtida através do método de Newton. A aplicação do método de Newton resulta no sistema matricial, que, em sua forma simplificada, é representada por:

$$
W \Delta d=-\nabla L a
$$

onde

$$
W=\left(\begin{array}{cccccccc}
\nabla_{x x}^{2} L a & 0 & 0 & 0 & J_{1}(x)^{T} & I & I & J_{2}(x)^{T} \\
0 & \delta S u & 0 & 0 & 0 & I & 0 & 0 \\
0 & 0 & \delta S \ell & 0 & 0 & 0 & -I & 0 \\
0 & 0 & 0 & \delta S & 0 & 0 & 0 & I \\
J_{1}(x) & 0 & 0 & 0 & 0 & 0 & 0 & 0 \\
I & I & 0 & 0 & 0 & 0 & 0 & 0 \\
I & 0 & -I & 0 & 0 & 0 & 0 & 0 \\
J_{2}(x) & 0 & 0 & I & 0 & 0 & 0 & 0
\end{array}\right)
$$

é a matriz Hessiana da função Lagrangiana com 


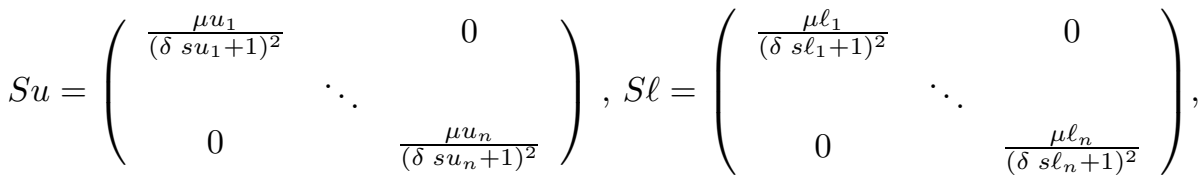

$$
\begin{aligned}
& S=\left(\begin{array}{ccc}
\frac{\mu_{1}}{\left(\delta s_{1}+1\right)^{2}} & & 0 \\
& \ddots & \\
0 & & \frac{\mu_{r}}{\left(\delta s_{r}+1\right)^{2}}
\end{array}\right) \text { e } \\
& \nabla_{x x}^{2} L a=\nabla_{x x}^{2} f(x)+\sum_{i=1}^{m} \lambda_{i} \nabla_{x x}^{2} g_{i}(x)+\sum_{j=1}^{r} \pi_{j} \nabla_{x x}^{2} h_{j}(x) ;
\end{aligned}
$$

$\Delta d^{T}=(\Delta x, \Delta s u, \Delta s \ell, \Delta s, \Delta \lambda, \Delta \pi u, \Delta \pi \ell, \Delta \pi)$ e $\nabla L a$ é dado em (3.6).

A Atualização das variáveis $x, s u, s \ell$ e $s$ e dos vetores Multiplicadores de Lagrange, $\lambda, \pi u, \pi \ell$ e $\pi$ é realizada da seguinte forma:

$$
\begin{aligned}
& x^{K+1}=x^{K}+\alpha_{p} \Delta x^{K} \\
& s u^{K+1}=s u^{K}+\alpha_{p} \Delta s u^{K} \\
& s \ell^{K+1}=s \ell^{K}+\alpha_{p} \Delta s \ell^{K} \\
& s^{K+1}=s^{K}+\alpha_{p} \Delta s^{K} \\
& \lambda^{K+1}=\lambda^{K}+\alpha_{d} \Delta \lambda^{K} \\
& \pi u^{K+1}=\pi u^{K}+\alpha_{d} \Delta \pi u^{K} \\
& \pi \ell^{K+1}=\pi \ell^{K}+\alpha_{d} \Delta \pi \ell^{K} \\
& \pi^{K+1}=\pi^{K}+\alpha_{d} \Delta \pi^{K},
\end{aligned}
$$

onde $\alpha_{p}$ e $\alpha_{d}$ são os passos utilizados na atualização das variáveis primais e duais, respectivamente. Uma sugestão para o cálculo do passo máximo é a estratégia utilizada por [6] e [10] dada por:

$$
\begin{gathered}
\alpha_{p}=\min \left\{\tau\left(\min _{\Delta s u<0} \frac{s u}{|\Delta s u|}, \min _{\Delta s \ell<0} \frac{s \ell}{|\Delta s \ell|}, \min _{\Delta s<0} \frac{s}{|\Delta s|}\right), 1\right\}, \\
\alpha_{d}=\min \left\{\tau\left(\min _{\Delta \pi u>0} \frac{-\pi u}{|\Delta \pi u|}, \min _{\Delta \pi \ell<0} \frac{\pi \ell}{|\Delta \pi \ell|}, \min _{\Delta \pi>0} \frac{-\pi}{|\Delta \pi|}\right), 1\right\},
\end{gathered}
$$

onde $\tau=0,9995$ é um valor determinado empiricamente e, que segundo [12], é derivada da fórmula $1-\frac{1}{9 \sqrt{p}}$, onde $p$ é o número de restrições do problema.

Os multiplicadores de Lagrange da Barreira Modificada $\mu u, \mu \ell$ e $\mu$ são atualizados utilizando-se a regra (2.5), encontrada em [8]:

$$
\begin{aligned}
\mu u_{k}^{K+1} & =\frac{\mu u_{k}^{K}}{\delta^{K} s u_{k}^{K}+1}, k=1, \ldots, n, \\
\mu \ell_{k}^{K+1} & =\frac{\mu \ell_{k}^{K}}{\delta^{K} s \ell_{k}^{K}+1}, k=1, \ldots, n, \\
\mu_{j}^{K+1} & =\frac{\mu_{j}^{K}}{\delta^{K} s_{j}^{K}+1}, j=1, \ldots, r,
\end{aligned}
$$


e o fator de barreira da seguinte forma:

$$
\left(\delta^{-1}\right)^{K+1}=\frac{\left(\delta^{-1}\right)^{K}}{\rho}, \rho>0,
$$

onde $\rho$ é denominado parâmetro de correção.

\subsection{Algoritmo}

O método da função Lagrangiana Barreira Modificada pode ser apresentado pelo seguinte algoritmo:

Passo inicial:

Dado o problema (3.1) construa a função Lagrangiana Barreira Modificada (3.4);

Faça $K=0$; Escolha uma solução inicial para as variáveis e fatores do problema: $x^{0}, s u^{0}, s \ell^{0}, s^{0}, \lambda^{0}, \pi u^{0}, \pi \ell^{0}, \pi^{0}, \mu u^{0}, \mu \ell^{0}, \mu^{0},\left(\delta^{-1}\right)^{0}$

Passo iterativo:

I1) Determine o sistema (3.7) e resolva-o;

I2) Atualize as variáveis $x, s u, s \ell, s, \lambda, \pi u, \pi \ell, \pi$ utilizando (3.8);

I3) Se o critério de parada para o método de Newton é satisfeito, vá para o passo I4); Senão, volte ao passo I1);

I4) Se as variáveis do problema satisfazem KKT, FIM; Senão, vá ao passo I5);

I5) Atualize os multiplicadores de Lagrange através de (3.11), (3.12) e (3.13) e o fator Barreira usando (3.14), faça $K=K+1$ e volte a I1).

\section{Resultados Numéricos}

O algoritmo foi implementado em linguagem FORTRAN, em um microcomputador Pentium III -600 MHz, com 128 Mbytes de memória RAM. A função objetivo utilizada foi perdas de potência ativa na transmissão para os sistemas elétricos de 3 e de 162 barras. Os dados do sistema de 162 barras são encontrados no sitio: (http://www.sor.princeton.edu/ rvdb/ampl/nlmodels/power/IEEE162a.bus). Esta função objetivo é não-linear, não convexa e não separável tornando o problema de difícil solução. Como critério de parada utilizamos as condições de KKT e a garantia da convergência na região factível a um erro máximo para as restrições de igualdade de $10^{-3}$. 


\subsection{Sistema Elétrico de 3 barras}

O problema de FPO, para o sistema elétrico de 3 barras, pode ser formulado como:

$$
\begin{aligned}
& \text { Minimizar } f\left(V_{1}, V_{2}, V_{3}, \theta_{2}, \theta_{3}\right)=4\left(V_{2}^{2}+V_{3}^{2}-2 V_{2} V_{3} \cos \left(\theta_{2,3}\right)\right)+ \\
& \quad 4\left(V_{3}^{2}+V_{1}^{2}-2 V_{3} V_{1} \cos \theta_{3}\right) \\
& \text { sujeito } a: \\
& \quad 1,7-\quad 4 V_{2}^{2}-V_{2} V_{3}\left(-4 \cos \left(\theta_{2,3}\right)-10 \operatorname{sen}\left(\theta_{2,3}\right)\right)=0, \\
& -2,0-8 V_{3}^{2}-V_{3} V_{2}\left(-4 \cos \left(\theta_{3,2}\right)-10 \operatorname{sen}\left(\theta_{3,2}\right)\right)-V_{3} V_{1}\left(-4 \cos \theta_{3}+5 \operatorname{sen} \theta_{3}\right)=0, \\
& -1,0-15 V_{3}^{2}-V_{3} V_{2}\left(-4 \operatorname{sen}\left(\theta_{3,2}\right)-10 \cos \left(\theta_{3,2}\right)\right)-V_{3} V_{1}\left(-4 \operatorname{sen} \theta_{3}+5 \cos \theta_{3}\right)=0, \\
& \quad 0,9 \leq V_{1} \leq 1,1, \\
& \quad 0,9 \leq V_{2} \leq 1,1, \\
& \quad 0,9 \leq V_{3} \leq 1,1,
\end{aligned}
$$

$\operatorname{com} \cos \left(\theta_{i, j}\right)=\cos \left(\theta_{i}-\theta j\right)$ e $\operatorname{sen}\left(\theta_{i, j}\right)=\operatorname{sen}\left(\theta_{i}-\theta j\right)$. O problema foi inicializado usando : $V_{1}=V_{2}=1,05, V_{3}=1,0, \theta_{2}=-0,0349, \theta_{3}=-0,873$, $\lambda_{1}=\lambda_{2}=\lambda_{3}=0, \mu \ell_{1}=\mu \ell_{2}=\mu \ell_{3}=1,0, \mu u_{1}=\mu u_{2}=\mu u_{3}=1,0$; o fator de barreira $\delta^{-1}=\frac{1}{43,52}$ e parâmetro de correção $\rho=1,15$; as variáveis auxiliares e os multiplicadores de Lagrange são determinados através das equações: $s u_{k}=V_{\max }-V_{k}$, $s \ell_{k}=-V_{\min }+V_{k}, \pi u_{k}=-\mu u_{k} /\left(\delta * s u_{k}+1\right)$ e $\pi \ell_{k}=\mu \ell_{k} /\left(\delta * s \ell_{k}+1\right), k=1, \ldots, 3$, $\operatorname{logo}, s u_{1}=s u_{2}=0,05, s \ell_{1}=s \ell_{2}=0,15, s u_{3}=s \ell_{3}=0, \pi u_{1}=\pi u_{2}=\pi u_{3}=0,05$, $\pi \ell_{1}=\pi \ell_{2}=\pi \ell_{3}=-0,05$. As tabelas 1,2 e 3 apresentam os valores das variáveis primais, função objetivo e variáveis duais, durante o processo de otimização.

Tabela 1 - Valores da FO e das variáveis durante o processo de otimização.

\begin{tabular}{cccccccccc}
\hline \hline IT & FO & $V_{1}$ & $V_{2}$ & $V_{3}$ & $\theta_{2}$ & $\theta_{3}$ & $s u_{1}$ & $s \ell_{1}$ & $s u_{2}$ \\
\hline \hline 1 & 0,134 & 1,036 & 1,081 & 0,960 & 0,094 & -0.018 & 0,064 & 0,136 & 0,019 \\
2 & 0,138 & 1,054 & 1,093 & 0,971 & 0,091 & $-0,017$ & 0,046 & 0,154 & 0,007 \\
\hline \hline
\end{tabular}

Tabela 2 - Valores das variáveis durante o processo de otimização.

\begin{tabular}{cccccccccc}
\hline \hline IT & $s \ell_{2}$ & $s u_{3}$ & $s \ell_{3}$ & $\pi u_{1}$ & $\pi \ell_{1}$ & $\pi u_{2}$ & $\pi \ell_{2}$ & $\pi u_{3}$ & $\pi \ell_{3}$ \\
\hline \hline 1 & 0,181 & 0,140 & 0,060 & 0,254 & $-0,144$ & 0,448 & $-0,109$ & 0,126 & $-0,248$ \\
2 & 0,193 & 0,129 & 0,071 & 0,065 & $-0,018$ & 0,328 & $-0,011$ & 0,019 & $-0,065$ \\
\hline \hline
\end{tabular}

Tabela 3 - Valores das variáveis durante o processo de otimização.

\begin{tabular}{cccccccccc}
\hline \hline IT & $\mu u_{1}$ & $\mu \ell_{1}$ & $\mu u_{2}$ & $\mu \ell_{2}$ & $\mu u_{3}$ & $\mu \ell_{3}$ & $\lambda_{1}$ & $\lambda_{2}$ & $\lambda_{3}$ \\
\hline \hline 1 & 0,264 & 0,145 & 0,544 & 0,113 & 0,141 & 0,277 & $-0,027$ & 0,096 & 0,069 \\
2 & 0,080 & 0,017 & 0,402 & 0,011 & 0,019 & 0,061 & $-0,035$ & 0,089 & 0,075 \\
\hline \hline
\end{tabular}

O estado final do sistema de 3 barras apresenta todas as variáveis dentro de seus limites e a solução final obedeceu às restrições do sistema, satisfazendo KKT a uma precisão de $10^{-3}$. O tempo de CPU medido, neste caso foi nulo. Como o sistema é muito pequeno a função utilizada para medir o tempo computacional não conseguiu registrar o valor, devido ao tempo ser pouco significativo. 


\subsection{Sistema Elétrico de 162 barras}

O problema de FPO associado ao sistema elétrico de 162 barras tem a mesma estrutura do sistema de 3 barras e é composto por uma função objetivo, 311 restrições de igualdade, 432 restrições de desigualdade. Após aplicação da nova abordagem trabalhamos com um total de 1974 variáveis e uma matriz Hessiana da função Lagrangiana de ordem 1541.

O sistema elétrico de 162 barras possui as seguintes características: uma barra de geração, 11 barras de controle de reativo, 150 barras de carga, 280 linhas de transmissão e 43 transformadores com taps variáveis.

As condições iniciais do problema foram obtidas do sítio:

(http://www.sor.princeton.edu/ rvdb/ampl/nlmodels/power/IEEE162a.bus). O valor inicial do fator de barreira foi $\delta^{-1}=\frac{1}{40,0}$ e das variáveis $s u, s \ell, \pi u$ e $\pi \ell$ foram calculados como no sistema de 3 barras. O parâmetro de correção da barreira foi $\rho=1,6$. A Figura 1 apresenta a convergência do sistema de 162 barras.

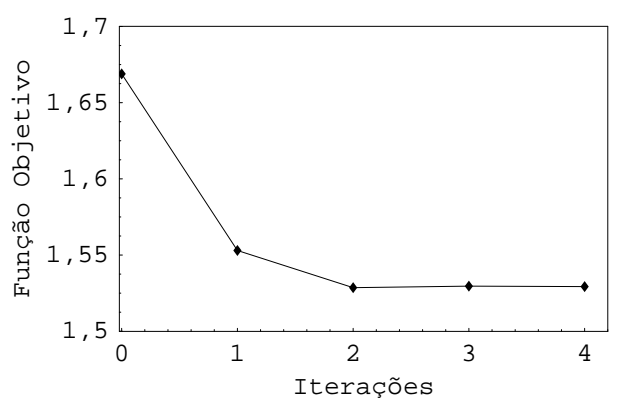

Figura 1: Convergência do Sistema Elétrico de 162 barras

O estado final do sistema de 162 barras apresenta todas as variáveis dentro de seus limites e a solução final obedeceu às restrições do sistema, satisfazendo KKT a uma precisão de $10^{-3}$ e o tempo computacional foi de 1,32 segundos sem considerar o tempo de leitura e impressão dos dados.

\section{Conclusão}

O artigo apresenta um método de resolução de problemas não-lineares e não convexos baseados na metodologia de pontos interiores. Neste método, as condições necessárias de primeira ordem são aplicadas à função Lagrangiana Barreira Modificada resultando num sistema não-linear, cuja solução é determinada através da utilização do método de Newton. Uma das vantagens deste método é que o fator de barreira não tende ao infinito quando o ótimo se aproxima. Os resultados numéricos apresentados neste trabalho evidenciam o potencial desta metodologia para a resolução do problema de FPO. O número de iterações do método está diretamente ligado à escolha dos fatores iniciais de barreira e seus respectivos parâmetros de 
correção. O método exige uma experiência prévia do sistema que será resolvido para um adequado ajuste do fator de barreira e de seu parâmetro de correção.

Abstract. A new approach to solve the Optimal Power Flow problem is described. We make use of some recent researches, especially in the area of interior point methods. In this approach, equality constraints are handled by Newton's method and inequality constraints by the modified barrier method. The numerical tests show the effective performance of this algorithm.

\section{Referências}

[1] J.L. Carpentier, Contribution a l'etude du dispatching economique, Bull. Soc. Fr. Elec., Ser. B3 (1962), 431-447.

[2] C.W. Carrol, The created response surface technique for optimizing nonlinear restrained systems, Operations Research, 9 (1961), 169-184.

[3] G.R.M. Costa, Optimal reactive dispatch through primal-dual method, IEEE Transactions on Power Systems, 12, No. 2 (1997), 669-674.

[4] A.V. Fiacco e G.P. McCormick, "Nonlinear Programming: Sequential Unconstrained Minimization Techniques", John Wiley-Sons, New York, 1968.

[5] K.R. Frisch, "The Logarithmic Potential Method of Convex Programming", University Institute of Economics (manuscript), Oslo, Norway, 1955.

[6] S. Granville, Optimal reactive dispatch through interior point methods, IEEE Transactions on Power Systems, 9 (1994), 136-146.

[7] N. Karmarkar, A new polynomial-time algorithm for linear programming, Combinatorics, 4 (1984), 373-395.

[8] R. Polyak, Modified barrier functions, Mathematical Programming, 54, No. 2 (1992), 177-222.

[9] D.F. Shanno e R.J. Vanderbei, Interior-point for nonconvex nonlinear programming, Mathematical Programming, 87 (2000), 303-316.

[10] G.L. Torres e V.H. Quintana, Optimal power flow in rectangular form via an interior point method, IEEE Transactions on Power Systems, 13, No. 4 (1998), 1211-1218.

[11] R.J. Vanderbei e D.F. Shanno, An interior-point algorithm for nonconvex nonlinear programming, Computational Optimization and Applications, 13 (1999), 231-252.

[12] M.H. Wright, Why a pure primal Newton barrier step may be infeasible, SIAM Journal on Optimization, 5, No. 1 (1995), 1-12. 